\title{
Conceptos actuales sobre la clasificación clínica y alteraciones electrofisiológicas en la disfunción del nódulo sinusal
}

\author{
José F. Alderete ${ }^{1}$, Osmar Antonio Centurión ${ }^{1,2}$ \\ ${ }^{1}$ Universidad Nacional de Asunción, Hospital de Clínicas, División de Medicina Cardiovascular. San Lorenzo. \\ Paraguay \\ ${ }^{2}$ Sanatorio Metropolitano, Departamento de Investigación en Ciencias de la Salud. Fernando de la Mora. Paraguay
} Cómo referenciar este artículo/
How to reference this article:
Alderete JF, Centurión OA. Conceptos actuales sobre la clasificación clínica y alteraciones electrofisiológicas en la disfunción del nódulo sinusal. Mem. Inst. Investig. Cienc. Salud. 2018; 16(2): 113-122

\section{RE S U M E N}

La disfunción del nódulo sinusal (DNS) es generalmente secundaria a la senescencia del nodo sinusal y del miocardio auricular circundante. Los pacientes con este trastorno son a menudo añosos y en general presentan otras comorbilidades. Los pacientes a menudo buscan atención médica con síntomas de aturdimiento, pre-síncope, síncope y, en pacientes con periodos alternantes de bradicardia y taquicardia, palpitaciones u otros síntomas asociados con una frecuencia cardíaca rápida. Debido a que los síntomas pueden ser de naturaleza variable, inespecíficos y frecuentemente transitorios, a veces puede ser difícil establecer esta relación síntoma-alteración electrocardiográfica. Los hallazgos electrocardiográficos típicos son uno o más episodios de bradicardia sinusal extrema (Rubenstein Tipo I), o pausas sinusales, paro y bloqueo de salida sinoatrial (Rubenstein Tipo II), o episodios de bradicardia y/o pausas alternantes con taquiarritmias auriculares (Rubenstein Tipo III). Las investigaciones basadas en el registro de electrogramas locales auriculares anormalmente prolongados y fraccionados durante el ritmo sinusal y su distribución característica en la aurícula derecha de pacientes con DNS han aportado un conocimiento importante sobre las propiedades electrofisiológicas de la aurícula patológica. El electrograma auricular anormal traduce una conducción auricular irregular, caracterizada por una actividad eléctrica local no homogénea, relacionada con una conducción anisotrópica, no uniforme y retardada a través de un miocardio auricular patológico, en el que se pueden originar arritmias por reentrada. La detección de electrogramas auriculares anormales en la DNS identifica a un grupo de pacientes con vulnerabilidad auricular aumentada y con una incidencia significativamente mayor de episodios espontáneos o inducidos de fibrilación auricular.

Palabras clave: disfunción del nódulo sinusal, síndrome de enfermedad sinusal, fibrilación auricular paroxística, vulnerabilidad auricular, electrogramas auriculares endocárdicos.

\section{Current concepts about the clinical classification and electrophysiological alterations in the sinus node dysfunction}

\section{A B S T R A C T}

Sinus node dysfunction (SND) is often secondary to senescence of the sinus node and surrounding atrial myocardium. Patients with this disorder are frequently elderly and generally have other comorbidities. Patients with SND often seek medical attention with symptoms of lightheadedness, presyncope, syncope, and, in patients with alternating periods of bradycardia and tachycardia, palpitations and/or other symptoms associated with 
a rapid heart rate. Because symptoms may be variable in nature, nonspecific, and frequently transient, it may be challenging at times to establish this symptom-rhythm relationship. Typical electrocardiographic findings are one or more episodes of extreme sinus bradycardia (Rubenstein type I), or sinus pauses, arrest, and sinoatrial exit block (Rubenstein type II), or alternating bradycardia and atrial tachyarrhythmias (Rubenstein type III). Investigations based on the recording of abnormally prolonged and fractionated local atrial electrograms during sinus rhythm and their characteristic distribution in the right atrium of patients with SND have provided important knowledge about the pathological atrium electrophysiological properties. Abnormal atrial electrogram results in an irregular atrial conduction, characterized by a non-homogeneous local electrical activity, related to an anisotropic, non-uniform and delayed conduction through a pathological atrial myocardium, in which reentry arrhythmias may arise. Abnormal atrial electrograms detection in SND identifies a group of patients with increased atrial vulnerability and a significantly higher incidence of spontaneous or induced episodes of atrial fibrillation.

Keywords: sinus node dysfunction, sick sinus syndrome, paroxysmal atrial fibrillation, atrial vulnerability, endocardial atrial electrograms.

\section{INTRODUCCIÓN}

El síndrome del seno enfermo, término acuñado por Lown para describir las alteraciones del ritmo cardiaco después de la cardioversión de corriente continua de la fibrilación auricular crónica, se caracteriza por una disfunción crónica del nodo sinusal que a menudo es secundaria a la senescencia del nodo sinusal y al miocardio auricular circundante ${ }^{(1-5)}$. Actualmente se denomina disfunción del nódulo sinusal (DNS) a esta entidad nosológica. Los pacientes con este trastorno son a menudo añosos (aunque algunas publicaciones describen una distribución bimodal) y en general presentan otras comorbilidades ${ }^{(6-10)}$.

Las anomalías electrocardiográficas observadas (bradicardia, pausas sinusales, paro sinusal), frecuentemente se alternan con episodios paroxísticos de taquiarritmias supraventriculares ${ }^{(2,8)}$. Los pacientes a menudo buscan atención médica con síntomas de aturdimiento, presíncope, síncope y, en pacientes con periodos alternantes de bradicardia y taquicardia, palpitaciones u otros síntomas asociados con una frecuencia cardíaca rápida ${ }^{(3-}$ 5). Debido a que los síntomas pueden ser de naturaleza variable, inespecíficos y frecuentemente transitorios, a veces puede ser difícil establecer esta relación síntomaalteración electrocardiográfica ${ }^{(8-10)}$. Los hallazgos electrocardiográficos típicos son uno o más episodios de bradicardia sinusal extrema (Rubenstein Tipo I), o pausas sinusales, paro y bloqueo de salida sinoatrial (Rubenstein Tipo II), o episodios de bradicardia y/o pausas alternantes con taquiarritmias auriculares (Rubenstein Tipo III $)^{(2)}$. Justamente, son estos hallazgos los que nos llevan a realizar esta revisión y a analizar detalladamente en este manuscrito ciertos conceptos actuales sobre la clasificación clínica y alteraciones electrofisiológicas en la disfunción del nódulo sinusal que nos conduce a poder determinar quiénes son los pacientes con DNS que son más susceptibles de desarrollar episodios sostenidos de fibrilación auricular paroxística.

\section{Conceptos Fisiopatológicos y Clasificación Clínica}

Se han propuesto 2 mecanismos principales para explicar la bradicardia en la disfunción del nódulo sinusal. En primer lugar, podría tratarse de una alteración en la generación de los impulsos dentro del nodo sinusal; en segundo lugar, un trastorno de la conducción de los impulsos desde el nódulo sinusal hacia la aurícula ${ }^{(3)}$. Los factores causantes podrían ser intrínsecos (enfermedades que alteran directamente el nodo sinusal) o extrínsecos. La causa más frecuente es la fibrosis degenerativa idiopática que se asocia a edad avanzada ${ }^{(4)}$. Podemos observar las causas más frecuentes de la DNS en la Tabla 1.

En 1972, Rubenstein et al. ${ }^{(2)}$ realizaron una revisión bastante descriptiva desde el punto de vista clínico de 56 pacientes con episodios de bradicardia documentada. La serie incluyó a 31 mujeres y 25 hombres con edades comprendidas entre los 26 y los 90 años, con una edad media de 65 años. Con el objeto de clasificar a los pacientes de acuerdo a los hallazgos electrocardiográficos, los dividieron en 3 grupos $^{(2)}$ :

Grupo I: 8 pacientes con bradicardia sinusal extrema inexplicada (menos de 50 latidos/minuto, y frecuentemente menor a 40).

Grupo II: 15 pacientes con al menos 1 episodio documentado de paro sinusal o bloqueo sinoauricular y con latidos de escape de la unión o ventriculares. 
Grupo III: 33 pacientes con el síndrome de bradicardia-taquicardia (alternancia de bradicardia y taquiarritmias auriculares paroxísticas). Las taquiarritmias más frecuentes fueron la fibrilación auricular, el flutter auricular y las taquicardias auriculares paroxísticas, aunque muchos pacientes exhibieron una combinación de estas arritmias auriculares, es decir, más de un tipo de taquiarritmia en diferentes momentos de su evolución clínica ${ }^{(2)}$.

Tabla 1. Causas más frecuentes como mecanismo de producción de Disfunción del Nódulo Sinusal

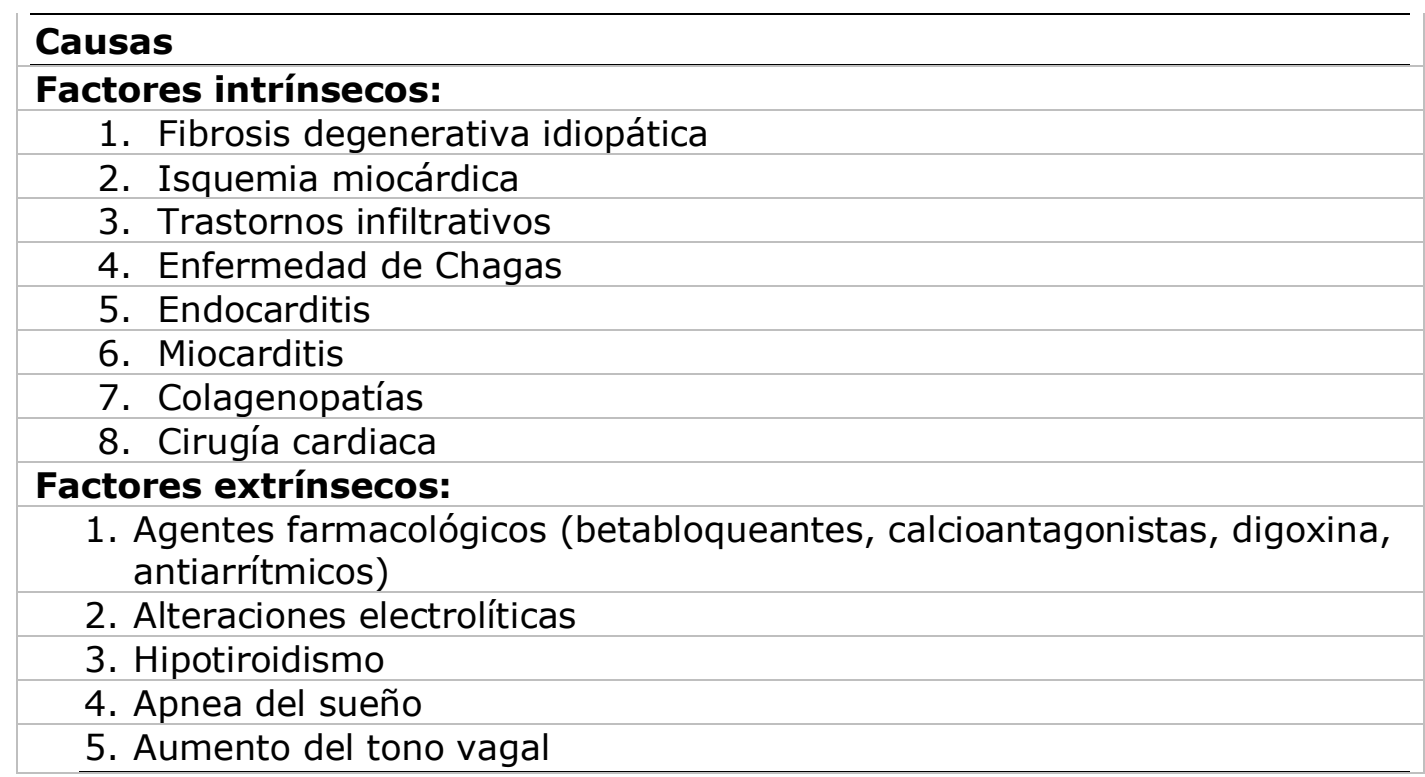

\section{Diagnóstico clínico y electrofisiológico en la disfunción del nódulo sinusal}

No existen criterios estandarizados para establecer el diagnóstico de DNS. La clave para llegar al diagnóstico de esta entidad es establecer una correlación entre los síntomas clínicos descritos por los pacientes y los hallazgos electrocardiográficos. Un electrocardiograma convencional de rutina o un monitoreo Holter de 24 horas pueden confirmar el diagnóstico si los hallazgos electrocardiográficos típicos pueden correlacionarse con síntomas. Los hallazgos electrocardiográficos típicos son uno o más episodios de bradicardia sinusal extrema (Rubenstein Tipo I), o pausas sinusales, paro y bloqueo de salida sinoatrial (Rubenstein Tipo II), o episodios de bradicardia y/o pausas alternantes con taquiarritmias auriculares (Rubenstein Tipo III) ${ }^{(2)}$. Sin embargo, en algunos pacientes, es posible que se requieran pruebas diagnósticas adicionales y no se debe diagnosticar la DNS hasta que se hayan identificado y tratado las causas potencialmente reversibles, que incluyen el uso de fármacos, isquemia miocárdica, hipotiroidismo y desequilibrio autonómico. Asimismo, los atletas bien entrenados presentan frecuencias cardiacas bastante bajas que hay que tener en consideración. Si el electrocardiograma y el monitoreo Holter de 24 horas repetido no logran documentar la causa de los síntomas de un paciente, debe considerarse la posibilidad de utilizar dispositivos de registro de eventos externo o de un monitor cardiaco implantable de registro continuo, un loop-recorder ${ }^{(5)}$.

Las pruebas de esfuerzo pueden ayudar a identificar la función anormal del nódulo sinusal, excluir la isquemia miocárdica, y pueden ayudar a guiar la programación del dispositivo para los pacientes que finalmente reciben un marcapasos permanente. La incompetencia cronotrópica, es decir, un aumento subnormal en la frecuencia cardíaca después del ejercicio puede ayudar a identificar a las personas con función anómala del nódulo sinusal que pueden beneficiarse de la implantación de un marcapasos ${ }^{(6)}$. Los estudios electrofisiológicos permiten determinar ciertos parámetros que hablan de la DNS como por ejemplo el tiempo de recuperación del nódulo sinusal, y el tiempo de conducción del nódulo sinusal. Por lo tanto, este estudio invasivo podría considerarse especialmente en aquellos pacientes que persisten sintomáticos y en quienes no se han documentado las alteraciones electrocardiográficas descritas ${ }^{(7)}$.

Los estudios electrofisiológicos en pacientes con disfunción del nódulo sinusal Tipo III de Rubenstein presentan ciertas limitaciones. Debido a la facilidad con que estos pacientes 
desarrollan fibrilación auricular mediante la estimulación cardíaca programada durante el estudio electrofisiológico en el laboratorio, no se puede realizar un análisis detallado de las propiedades electrofisiológicas del miocardio auricular ya que la fibrilación auricular no permite la realización de las mediciones correspondientes. Por ende, los datos obtenidos mediante el mapeo endocárdico auricular durante ritmo sinusal adquieren una incuestionable importancia en el conocimiento del sustrato electrofisiológico auricular. Las investigaciones basadas en el registro de electrogramas locales auriculares anormalmente prolongados y fraccionados durante el ritmo sinusal y su distribución característica en la aurícula derecha de pacientes con disfunción del nódulo sinusal han aportado un conocimiento importante sobre las propiedades electrofisiológicas de la aurícula patológica. Hemos demostrado previamente que los electrogramas auriculares endocárdicos anormalmente prolongados y fraccionados registrados en la aurícula derecha denotan una conducción auricular irregular y que se registran frecuentemente en pacientes con disfunción del nódulo sinusal Tipo III de Rubenstein. El mapeo endocárdico auricular en pacientes con fibrilación auricular paroxística ha proporcionado más conocimiento acerca del sustrato electrofisiológico de esta taquiarritmia. La detección de electrogramas auriculares anormales identifica a un grupo de pacientes con vulnerabilidad auricular aumentada y con incidencia significativamente mayor de episodios espontáneos o inducidos de fibrilación auricular.

\section{Alteraciones miocárdicas de la aurícula derecha en el mapeo endocárdico}

Ya desde los inicios del estudio electrofisiológico como procedimiento de diagnóstico invasivo, la técnica de mapeo endocárdico auricular ha sido empleada para realizar un detallado análisis de la secuencia de activación miocárdica y ha contribuido a un mejor entendimiento de los mecanismos arritmogénicos en pacientes con taquicardias supraventriculares ${ }^{(11-13)}$. El mapeo endocárdico por catéter de la aurícula derecha durante el ritmo sinusal ha permitido registrar electrogramas auriculares anormales de ciertas zonas auriculares patológicas, en pacientes con disfunción del nódulo sinusal ${ }^{(14-16)}$. La técnica del mapeo endocárdico con catéter de la aurícula derecha permite registrar los electrogramas bipolares endocárdicos de las paredes anterior, posterior, lateral y medial de las zonas, alta, media y baja de la aurícula derecha (Figura 1).

Figura 1: Sitios de mapeo endocárdico por catéter de la aurícula derecha. Los electrogramas auriculares fueron registrados de 12 sitios endocárdicos en cada paciente. Los sitios que figuran de la A a la D corresponden a la parte alta de la aurícula derecha mientras que los sitios de la $\mathrm{E}$ a la $\mathrm{H}$ son de la parte media, y aquellos de la I a la $\mathrm{L}$ son de la parte baja. Los sitios marcados como A, E y I corresponden a la región anterior, los sitios $B, F$ y $\mathrm{J}$ a la región lateral, los sitios $\mathrm{C}, \mathrm{G}$ y $\mathrm{K}$ a la región posterior, y los sitios $\mathrm{D}, \mathrm{H}$ y L corresponden a la región medial de la aurícula derecha. SVC: vena cava superior. IVC: vena cava inferior. Ao: Aorta. PA: arteria pulmonar. LA: aurícula izquierda. RV: ventrículo derecho. LV: ventrículo izquierdo. En la parte inferior (A) se observa un electrograma auricular endocárdico anormal con una duración de $130 \mathrm{~ms}$ y 10 deflecciones fragmentadas. (B) demuestra un electrograma auricular endocárdico normal que tiene una duración de 80 ms y 2 deflecciones fragmentadas. Reimpreso con permiso de Centurion et al. Influence of advancing age on fractionated right atrial endocardial electrograms. Am J Cardiol 2005;96:239-242.
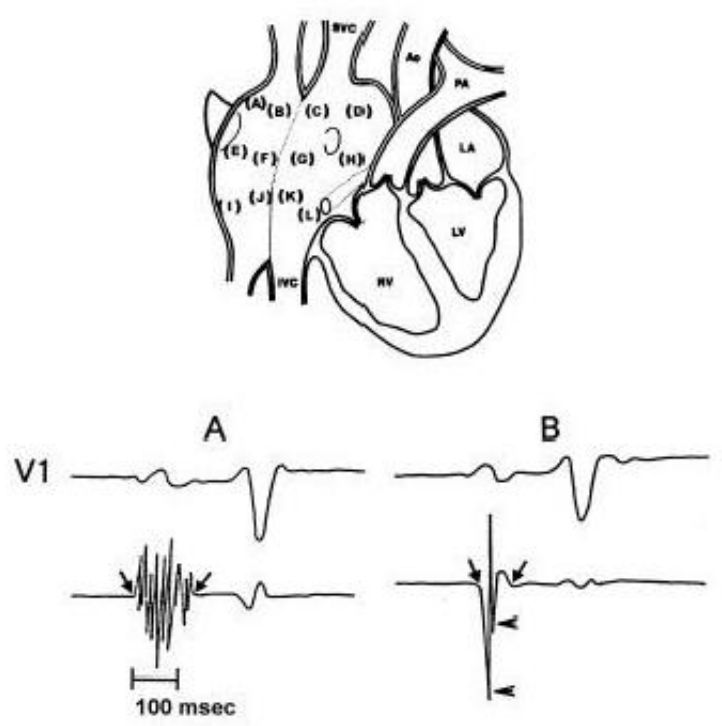

Mediante esta técnica diseñada e implementada por nuestro laboratorio del Departamento de Cardiología de la Universidad de Nagasaki, Japón, ${ }^{(17-23)}$ se puede registrar doce electrogramas endocárdicos en los diferentes sitios de la aurícula derecha y en cada uno de ellos se midieron cuantitativamente la duración y el número de deflexiones fragmentadas. La definición de la duración de un electrograma auricular es el intervalo 
desde el comienzo de la actividad eléctrica más temprana que se desvía de la línea de base hasta la última deflexión del electrograma auricular donde la línea de base cruza la línea isoeléctrica de la actividad auricular. El número de deflexiones fragmentadas se obtiene mediante el conteo de las deflexiones que apuntan hacia abajo como podemos observar en la Figura 2.

Figura 2: Ejemplos de un mismo electrograma auriculares endocárdico registrados durante ritmo sinusal para demostrar la medición de la duración del electrograma (parte superior) y del número de deflecciones fragmentadas del electrograma (parte inferior). V1: es la derivación electrocardiográfica. RA: es el electrograma auricular endocárdico de la aurícula derecha. Reimpreso con permiso de Konoe et al. Electrophysiological abnormalities of the atrial muscle in patients with manifest Wolff-Parkinson-White syndrome associated with paroxysmal atrial fibrillation. PACE 1992;15:1040-1052.

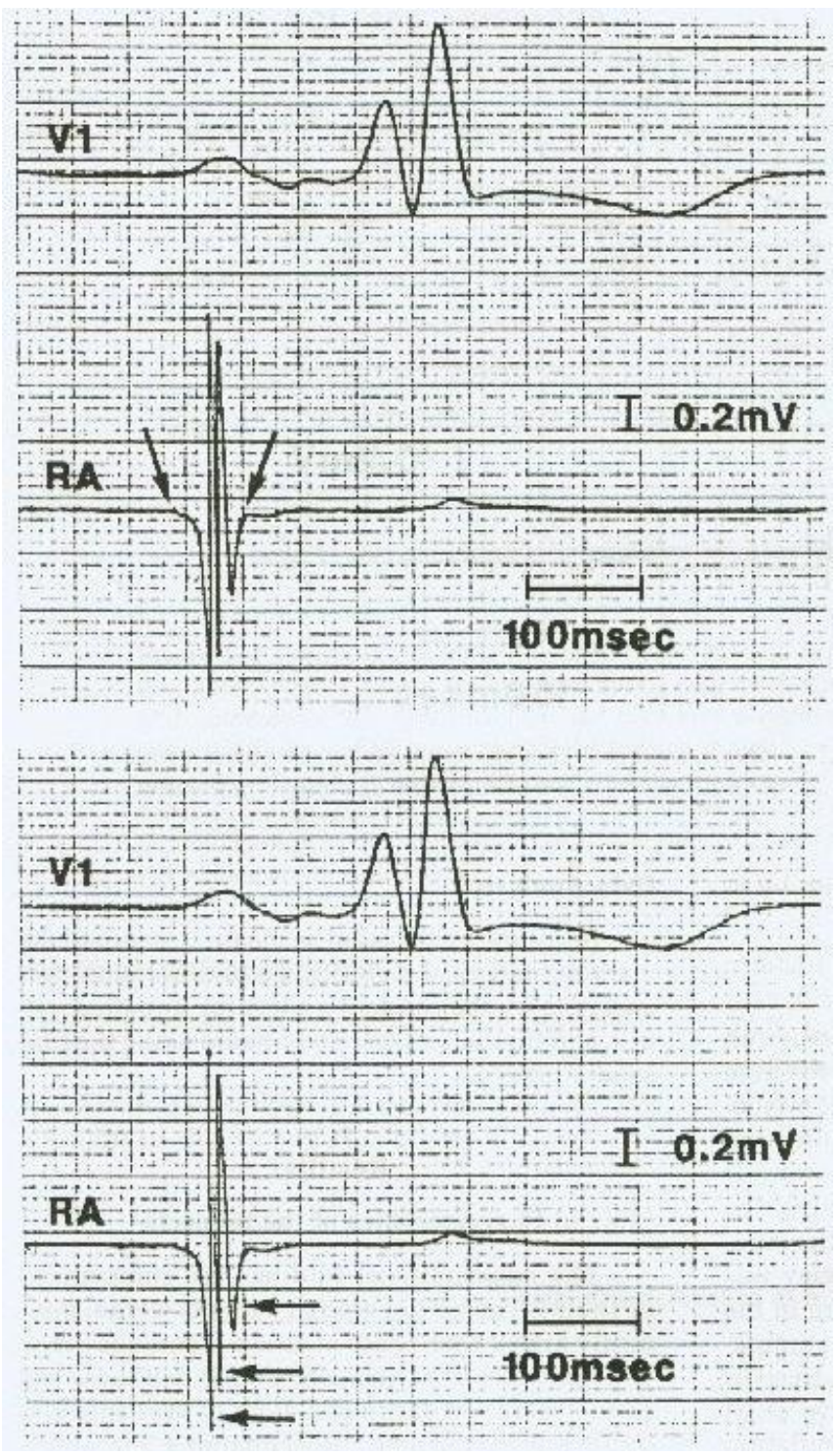

Tanigawa et al. definieron los valores normales de los electrogramas auriculares endocárdicos basados en las medidas cuantitativas y cualitativas de 516 electrogramas endocárdicos en 43 sujetos con función normal del nódulo sinusal y sin fibrilación auricular paroxística ${ }^{(18)}$. La duración promedio y el número promedio de deflexiones fragmentadas de los electrogramas auriculares endocárdicos de estos pacientes fue de $74 \pm 11 \mathrm{~ms}$ y $3,9 \pm 1,3$ (desvíos estándar) respectivamente. Por lo tanto, con estos valores promedios obtenidos en sujetos normales, se definieron como valores anormales a aquellos valores mayores a 2 desviaciones estándar ${ }^{(18)}$. Por ende, un electrograma auricular endocárdico anormal fue definido como aquel que tuviera una duración igual o mayor a $100 \mathrm{~ms}$ y/u ocho o más deflexiones fragmentadas (Figura 3). 
Figura 3: Se observa un electrograma auricular endocárdico anormal registrado en la parte lateral alta de la aurícula derecha. Reimpreso con permiso de Konoe A, Fukatani $M$, Tanigawa $M$, et al. Electrophysiological abnormalities of the atrial muscle in patients with manifest Wolff-ParkinsonWhite syndrome associated with paroxysmal atrial fibrillation. PACE 1992;15:1040-1052.

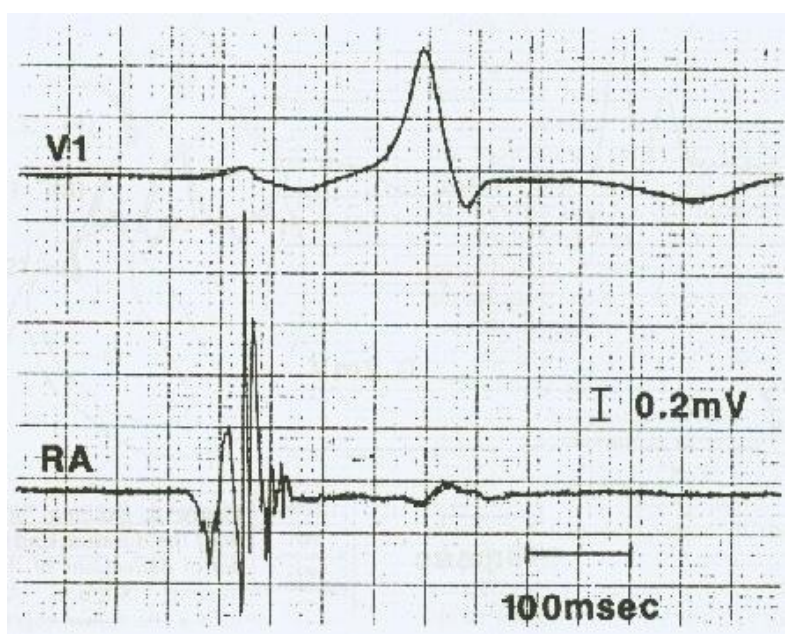

En condiciones tisulares normales pueden ocurrir algunos trastornos leves en la conducción de los impulsos cardiacos. Durante la conducción de un impulso eléctrico, la corriente axial fluye de una célula miocárdica a la adyacente a través de los discos intercalares, los cuales poseen normalmente una resistencia relativamente baja ${ }^{(24)}$. La conducción perpendicular al eje longitudinal de las fibras musculares auriculares puede ser retardada debido a que en dicha dirección la resistencia axial efectiva es mayor ${ }^{(24)}$. Esta mayor resistencia axial resulta en parte por la cantidad escasa y la longitud corta de los discos intercalares en la dirección latero-lateral de las fibras musculares, comparados con la dirección termino-terminal. Cuando las fibras musculares auriculares se encuentran separadas por tejido conjuntivo, las propiedades de conducción pueden alterarse debido a los efectos sobre la resistencia axial, creando una propagación discontinua y anisotrópica(2431). Al respecto, Spach et al. han demostrado que el enlentecimiento de la velocidad de conducción ocasiona una disminución en la amplitud y un aumento en la duración del electrograma extracelular del sistema de Purkinje canino(26). Para investigar la causa del aumento en la duración y en las deflecciones del electrograma, se ha estudiado y demostrado en un modelo computarizado de generación de electrogramas que la velocidad de conducción disminuida era responsable del aumento en la duración del electrograma, mientras que la resistencia intracelular aumentada, era la responsable de la naturaleza fraccionada del electrograma ${ }^{(30)}$.

Estudios patológicos detallados y cuantitativos realizados en pacientes con disfunción del nódulo sinusal Tipo III de Rubenstein han demostrado una fibrosis extensa del miocardio auricular en la vecindad del nódulo sinusal y en los tractos internodales ${ }^{(32-36)}$. Por lo tanto, un electrograma endocárdico auricular anormalmente prolongado y fraccionado registrado en estos pacientes mediante el mapeo de la aurícula derecha durante ritmo sinusal, podría traducir una actividad eléctrica localizada y no homogénea relacionada con una conducción retardada, no uniforme y anisotrópica a través de un miocardio auricular patológico ${ }^{(14-17)}$. Además, se ha demostrado histológicamente que los tejidos donde se originan los electrogramas anormalmente prolongados y fraccionados presentan procesos fibrodegenerativos ${ }^{(24-26)}$. Cuando las paredes auriculares se encuentran marcadamente alteradas por fibrosis, la onda de despolarización debe cambiar frecuentemente de dirección respecto de la orientación longitudinal de la fibra miocárdica. Esto provocaría bloqueos unidireccionales, conducción lenta y dispersión de los periodos refractarios en determinados sitios, generando los elementos fundamentales del mecanismo de la reentrada ${ }^{(24)}$.

Centurión $\mathrm{OA}$ et al. diseñaron un estudio para evaluar la relación existente entre ciertos parámetros electrofisiológicos que indican una vulnerabilidad auricular aumentada y los electrogramas auriculares anormales en pacientes con disfunción del nódulo sinusal ${ }^{(37)}$. Entre los indicadores electrofisiológicos de vulnerabilidad auricular aumentada fueron estudiadas la actividad auricular fragmentada, retardo en la conducción interauricular la respuesta auricular repetitiva y la fibrilación auricular sostenida, todas inducidas por estimulación auricular programada con extraestímulo simple (Figuras 4 y 5). Las figuras $1,2,3$ no se mencionan en el texto y ninguna figura está en el material salvo la leyenda de la Figura 1 que está parte en español y parte en inglés. En dicho trabajo se intentó esclarecer la importancia y el significado del registro de electrogramas auriculares anormales durante ritmo sinusal, en pacientes con disfunción del nódulo sinusal 
susceptibles de desarrollar fibrilación auricular. Demostraron que los pacientes que poseían electrogramas auriculares anormales tenían una vulnerabilidad auricular significativamente aumentada, comparados con aquellos que tenían electrogramas normales. Los electrogramas auriculares anormales mostraron una muy buena especificidad y valor predictivo positivo al evaluar la inducción de fibrilación auricular sostenida. La especificidad demostrada fue de $94 \%$ con un valor predictivo positivo del $93 \%{ }^{(37)}$.

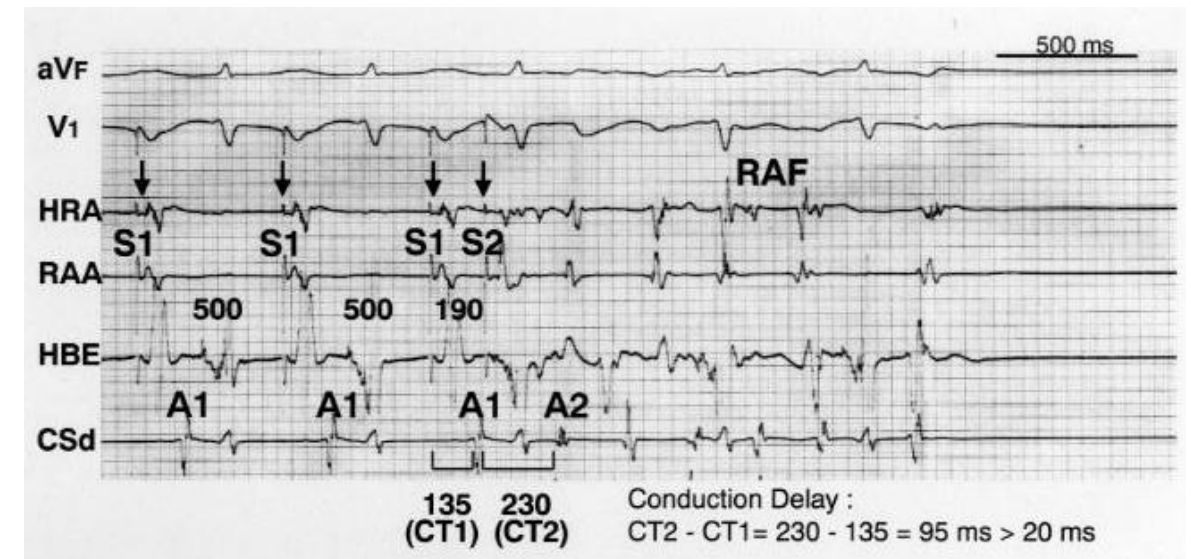

Figura 4: Estimulación auricular programada con extra-estímulo simple a un intervalo de acoplamiento de 190 ms con un intervalo de ciclo básico de $500 \mathrm{~ms}$ que genera una actividad auricular repetitiva (RAF) y un retardo en la conducción interauricular (CD). S1 y A1 se refiere al artefacto del estímulo y al electrograma auricular, respectivamente, del latido del ciclo básico. S2 y A2 se refiere al artefacto del estímulo y al electrograma auricular, respectivamente, del extra-estímulo. El intervalo S1-A1 a nivel del seno coronario distal (CSd) mide $135 \mathrm{~ms}$ (CT1). Sin embargo, el intervalo S2-A2 se prolonga a $230 \mathrm{~ms}$ (CT2) con el extra-estímulo. Por lo tanto, el retardo máximo en la conducción interauricular es de 95 ms (CT2-CT1) $(230-135=95)$. HRA: Parte lateral alta auricular. RAA: Apéndice auricular derecho. HBE: Electrograma del haz de His. CSd: Seno coronario distal. CT: Tiempo de conducción. Reimpreso con permiso de Isomoto et al. The effects of aging on the refractoriness and conduction of the atrium in patients with lone paroxysmal atrial fibrillation revealed with programmed atrial stimulation. Rev Soc Parag Cardiol 2005;3:25-30.

Figura 5: Se observa una estimulación auricular programada con extra-estímulo simple a un intervalo de acoplamiento (S1$\mathrm{S} 2)$ de $230 \mathrm{~ms}$ con un intervalo de ciclo básico $(B C L)$ de 500 ms que genera una actividad auricular fragmentada (FAA) y un retardo en la conducción interauricular (CD). El electrograma auricular de la parte alta de la aurícula derecha (HLRA) provocado por la estimulación del artefacto $\mathrm{S} 1$ mide $110 \mathrm{~ms}$ y se prolonga a $200 \mathrm{~ms}$ con la estimulación del artefacto S2. Reimpreso con permiso de Konoe et al. Electrophysiological abnormalities of the atrial muscle in patients with manifest Wolff-Parkinson-White syndrome associated with paroxysmal atrial fibrillation. PACE 1992;15:1040-1052.

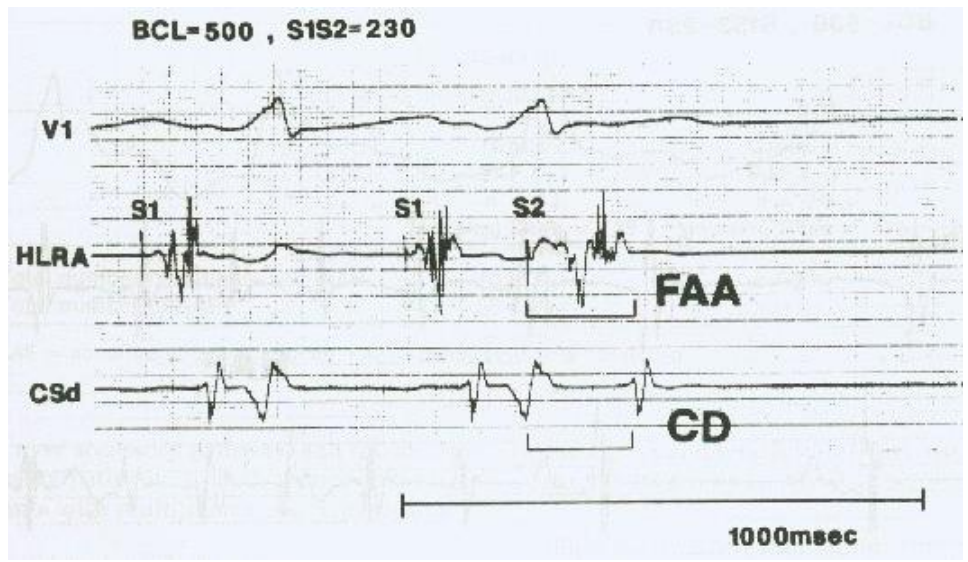

Si bien existen diversos factores que influyen en mayor o menor grado en la aparición de la fibrilación auricular en estos pacientes con disfunción del nódulo sinusal, los electrogramas auriculares anormales registrados durante ritmo sinusal en pacientes con alteraciones electrofisiológicas del miocardio auricular podrían ser considerados como indicadores de una vulnerabilidad auricular aumentada ${ }^{(37-42)}$.

La implicancia clínica demostrada es que la detección de electrogramas auriculares anormales durante ritmo sinusal en pacientes susceptibles de desarrollar fibrilación auricular, tales como aquellos con disfunción del nódulo sinusal, puede ayudar a identificar a un grupo de pacientes con vulnerabilidad auricular significativamente aumentada y con una incidencia significativamente mayor de episodios espontáneos o inducidos de fibrilación auricular paroxística ${ }^{(43-49)}$ ). Esto podría conducir a tomar las medidas preventivas necesarias para evitar las nefastas consecuencias de esta temible taquiarrítmia. 


\section{Manejo terapéutico y pronóstico en la disfunción del nódulo sinusal}

En primer lugar, se debe descartar y tratar las causas potencialmente reversibles. Además, se debe tener en cuenta a la bradicardia sinusal fisiológica. Los pacientes con una DNS que permanecen asintomáticos no requieren un tratamiento específico. La bradicardia sinusal sintomática es una indicación de estimulación con marcapasos ya que no existe tratamiento farmacológico eficaz para tratar este trastorno. Los síntomas pueden estar presentes en reposo, pero con mayor frecuencia se desarrollan durante el ejercicio.

El modo adecuado del marcapasos ha sido objeto de controversias. Los modos de marcapasos que preservan la sincronía auriculo-ventricular (AAIR o DDDR) son los recomendados actualmente en la guía actual de la Sociedad Europea de Cardiología ${ }^{(7)}$. El modo AAIR se reserva a aquellos pacientes con función preservada del nodo auriculoventricular (AV) y en quienes el riesgo de desarrollar disfunción del nodo AV es mínimo. En base a nuestra experiencia y observaciones electrofisiológicas y clínicas, hemos determinado que a la mayoría de los pacientes con DNS que poseen electrogramas auriculares anormales y una vulnerabilidad auricular aumentada, se les inserte un marcapasos bicameral programado en modo DVI. Los resultados iniciales de estudios prospectivos y de seguimiento a largo plazo avalan dicha conducta terapéutica.

Teniendo en cuenta que las taquiarritmias supraventriculares y los eventos tromboembólicos son frecuentes en los pacientes con DNS, se debe considerar el uso de anticoagulación oral y especialmente en aquellos pacientes con fibrilación auricular documentadas $^{(2,9)}$. La evolución de los pacientes con DNS es variable e impredecible. Los pacientes con antecedentes de síncope pueden presentar síncopes recurrentes ${ }^{(10)}$. La aparición de un bloqueo AV completo y de arritmias ventriculares malignas asociadas a los episodios de bradicardia es relativamente baja ${ }^{(2)}$. La incidencia de muerte súbita parece ser baja también y el tratamiento con marcapasos no mejora la supervivencia total, aunque mejora notablemente los síntomas y la calidad de vida. Además, permite utilizar fármacos para el manejo de las taquiarritmias $(2,7,10)$.

El pronóstico global de la DNS depende de varios factores, entre ellos: edad, comorbilidades, presencia simultánea de trastornos de conducción y fibrilación auricular que comporta un mayor riesgo de complicaciones tromboembólicas.

\section{CONCLUSIÓN}

Los hallazgos electrocardiográficos típicos son uno o más episodios de bradicardia sinusal extrema (Rubenstein Tipo I), o pausas sinusales, paro y bloqueo de salida sinoatrial (Rubenstein Tipo II), o episodios de bradicardia y/o pausas alternantes con taquiarritmias auriculares (Rubenstein Tipo III).

Las investigaciones basadas en el registro de electrogramas locales auriculares anormalmente prolongados y fraccionados durante el ritmo sinusal y su distribución característica en la aurícula derecha de pacientes con DNS han aportado un conocimiento importante sobre las propiedades electrofisiológicas de la aurícula patológica. El electrograma auricular anormal traduce una conducción auricular irregular, caracterizada por una actividad eléctrica local no homogénea, relacionada con una conducción anisotrópica, no uniforme y retardada a través de un miocardio auricular patológico, en el que se pueden originar arritmias por reentrada. La detección de electrogramas auriculares anormales en la DNS identifica a un grupo de pacientes con vulnerabilidad auricular aumentada y con una incidencia significativamente mayor de episodios espontáneos o inducidos de fibrilación auricular.

\section{REFERENCIAS BIBLIOGRÁFICAS}

1. Lown B. Electrical reversion of cardiac arrhythmias. Brit Heart J 1967;29 (4): 46989.

2. Rubenstein JJ, Schulman CL, Yurchak PM, DeSanctis RW. Clinical spectrum of the sick sinus syndrome. Circulation. 1972;46(1):513.

3. Ferrer MI. The sick sinus syndrome in atrial disease. JAMA. 1968;206(3):645-46.

4. Brignole M. Sick sinus syndrome. Clin Geriatr Med. 2002;18:211-27.

5. Jou CJ, Arrington CB, Barnett S, Shen J, Cho S, Sheng $X$, et al. A Functional Assay for Sick Sinus Syndrome Genetic Variants. Cell Physiol Biochem. 2017;42(5):2021-9. doi: $10.1159 / 000479897$.

6. Kay GN. Quantitation of chronotropic response: comparison of methods for ratemodulating permanent pacemakers. J Am Coll Cardiol. 1992;20(7):1533-41.

7. Brignole $M$, Auricchio A, Baron-Esquivias G, Bordachar P, Boriani G, Breithardt OA et al. 2013 ESC Guidelines on cardiac pacing and cardiac resynchronization therapy. The Task Force on cardiac pacing and resynchronization therapy of the European 
Society of Cardiology (ESC). Developed in collaboration with the European Heart Rhythm Association (EHRA). Eur Heart ] 2013;34: 2281-329.

8. Teeäär $T$, Serg $M$, Paapstel K, Kals J, Kals $M$, Zilmer $M$, et al. Heart rate reduction decreases central blood pressure in sick sinus syndromepatients with a permanent cardiac pacemaker. J Hum Hypertens 2018 ; 32:377-84. doi: 10.1038/s41371018-0051-4.

9. Narasimhan C, Sanyal J, Sethi R, Kothari Y, Malik FTN, Pandurangi $U$, et al. Underutilization of pacemaker therapy for sinus node dysfunction - Real world data from South Asia. Indian Heart J. 2017;69(5):607-12. Doi:1016/j.ihj.2017.02.013.

10. Benditt DG, Sakaguchi S, Lurie KG, Lu F. Sinus node dysfunction. En: Willerson J, Cohn J, Wellens JH, Holmes D, editors. Cardiovascular medicine. Nueva York: Springer;2007. 1925-41.

11. Menozzi C, Brignole $M$, Alboni $P$, Boni $L$, Paparella N, Gaggioli G, et al. The natural course of untreated sick sinus syndrome and identification of the variables predictive of unfavorable outcome. Am J Cardiol 1998;82:1205-9.

12. Amat-y-Leon $F$, Dhingra RC, Wu D, Denes P, Wyndham C, Rosen KM. Catheter mapping of retrograde atrial activation: observations during ventricular pacing and AV nodal reentrant paroxysmal tachycardia. Br Heart J 1976; 38: 355-62.

13. Kastor JA, Goldreyer BN, Moore EN, Shelburne JC, Manchester JH. Intraventricular conduction in man studied with an endocardial electrode catheter mapping technique: patients with normal QRS and right bundle branch block. Circulation 1975;51:786-96.

14. Centurión OA, Fukatani $M$, Shimizu $A$, Konoe $A$, Isomoto $S$, Tanigawa $M$ et al. Anterograde and retrograde decremental conduction over left-sided accessory atrioventricular pathways in the WolffParkinson-White syndrome. Am Heart J 1993; 125:1038-47.

15. Centurión OA, Fukatani $M$, Konoe $A$, Tanigawa $M$, Shimizu A, Isomoto $S$ et al. Different distribution of abnormal endocardial electrogram within the right atrium in patients with sick sinus syndrome. Br Heart J 1992;68:596-600.

16. Centurión OA, Isomoto $S$, Fukatani $M$, Shimizu A, Konoe A, Tanigawa $M$, et al. Relationship between atrial conduction defects and fractionated atrial endocardial electrograms in patients with sick sinus syndrome. PACE 1993;16:2022-33.

17. Centurión OA, Fukatani $M$, Konoe $A$, Tanigawa $M$, Shimizu A, Isomoto $S$, et al. Electrophysiological abnormalities of the atrial muscle in patients with sinus node dysfunction without tachyarrhythmias. Int J Cardiol 1992;37:41-50.

18. Hashiba $K$, Centurión $O A$, Shimizu A. The electrophysiological characteristics of the human atrial muscle in paroxysmal atrial fibrillation. Am Heart J 1996;131:778-98.

19. Tanigawa M, Fukatani M, Konoe A, Isomoto S, Kadena M, Hashiba K. Prolonged and fractioned right atrial electrograms during sinus rhythm in patients with paroxysmal atrial fibrillation and sick sinus node syndrome. J Am Coll Cardiol 1991;17:4038.

20. Centurión OA, Shimizu A, Isomoto $S$, Konoe $A$, Kaibara $M$, Hayano $M$, et al. Influence of advancing age on fractionated right atrial endocardial electrograms. Am J Cardiol 2005; 96:239-42.

21. Centurión OA, Isomoto $S$, Shimizu $A$, Konoe $A$, Yano $K$. The effects of aging on atrial endocardial electrograms in patients with paroxysmal atrial fibrillation. Clin Cardiol 2003;26:435-8.

22. Shimizu A, Centurión OA. Electrophysiological properties of the human atrial fibrillation. Cardiovasc Res 2002; 54:302-14.

23. Centurión OA, Isomoto $S$, Shimizu A. Electrophysiological changes of the atrium in patients with lone paroxysmal trial fibrillation. JAFIB 2010;1(12):656-62.

24. Centurión OA. Age-related electrophysiological changes of the atrial myocardium in patients with paroxysmal atrial fibrillation. J Cardiol Cardiol Curr Res 2015;3(6):00121. DOI: 10.15406.

25. Spach MS, Dolber PC. Relating extracellular potentials and their derivatives to anisotropic propagation at microscopic level in human cardiac muscle: Evidence for electrical uncoupling of side-to-side fiber connections with increasing age. Circ Res $1986 ; 58: 356-71$.

26. Garder PI, Ursel PC, Fenoglio JJ Jr, Wit AL. Electrophysiologic and anatomic basis for fractioned electrograms recorded from healed myocardial infarcts. Circulation 1985;72:596-611.

27. Savio-Galimberti E, Argenziano M, Antzelevitch C. Cardiac Arrhythmias Related to Sodium Channel Dysfunction. Handb Exp Pharmacol. 2017. doi: 10.1007/164_2017_43.

28. Spach MS, Miller WT, Geselowitz DB, Barr RC, Kootsey JM, Johnson EA. The discontinuous nature of propagation in normal canine cardiac muscle: Evidence for recurrent discontinuities of intracellular resistance that affect the membrane currents. Circ Res 1981;39:54.

29. Spach MS, Kootsey JM. The nature of electrical propagation in cardiac muscle. Am J Physiol: Heart and Circ Physiol 1983; 13:3-22.

30. Spach MS, Dolber PC, Heidlage JF. Interaction of inhomogeneities of 
repolarization with anisotropic propagation in dog atria: A mechanism for both preventing and initiating reentry. Circ Res 1989;65:1612-31.

31. Lesch MD, Spear JF, Simson MB. A computer model of the electrogram: What causes fractionation? J Electrocardiol 1988;21:S69-S73.

32. Spach MS, Miller III WT, Dolber PC, Kootsey JM, Sommer JR, Mosher CE Jr. The functional role of structural complexities in the propagation of depolarization in the atrium of the dog: Cardiac conduction disturbances due to discontinuities of effective axial resistivity. Circ Res 1982;50:171-91.

33. Sims BA. Pathogenesis of atrial arrhythmia. Br Heart J 1972;34:336-40.

34. Davis MJ, Pomerance A. Pathology of atrial fibrillation in man. Br Heart J 1972;34:5205.

35. Kaplan BM, Langendorf R, Lev M, Pick A. Tachycardia-bradycardia syndrome (socalled "sick sinus syndrome"): Pathology, mechanisms, and treatment. Am J Cardiol 1973;31:497-508.

36. Evans R, Shaw DB. Pathological studies in sinoatrial disorders (sick sinus syndrome). Br Heart J 1977;39:778-86.

37. Demoulin JC, Kulbertus HE. Histopathological correlates of sinoatrial disease. Br Heart J 1978;40:1384-9.

38. Centurión OA, Shimizu A, Isomoto $S$, Konoe A, Hirata $T$, Hano $O$, et al. Repetitive atrial firing and fragmented atrial activity elicited by extrastimuli in the sick sinus syndrome with and without abnormal atrial electrograms. Am J Med Sci 1994; 307(4):247-54.

39. Isomoto $S$, Fukatani $M$, Kone $A$, Tanigawa $M$, Centurión $O A$, Seto $S$, et al. The influence of advancing age on abnormal right atrial electrograms obtained by intraatrial catheter mapping during sinus rhythm in patients with paroxysmal atrial fibrillation. Jpn J Electroardiol 1992;12:21623.

40. Isomoto $S$, Shimizu $A$, Tanigawa $M$, Kaibara $M$, Centurión OA, Konoe $A$, et al. Effects of intravenous verapamil on atrial vulnerabilty. Jpn Circ J 1994;58:1-8.

41. Shimizu A, Kaibara $M$, Centurión OA, Kapuku G, Hirata T, Fukatani M, et al. Electrophysiologic effects of a new class III antiarrhythmic agent. E-4031, on the atrial flutter, atrial refractoriness, and conduction delay in a canine sterile pericarditis model. J Cardiovasc Pharm 1993;21:656-62.
42. Shimizu A, Fukatani $M$, Tanigawa $M$, Kaibara $M$, Konoe A, Centurión OA, et al. Mechanism of suppression of repetitive atrial firing by isoproterenol. Comparison with disopyramide. Int J Cardiol $1994 ; 43: 175-83$.

43. Isomoto $S$, Shimizu $A$, Konoe $A$, Kaibara $M$, Centurión OA, Fukatani $M$, et al. Electrophysiologic effect of E-4031, a new class III antiarrhythmic agent in patients with supraventricular tachyarrhythmias. Am J Cardiol 1993;71:1464-7.

44. Bao Z, Chen H, Yang B, Shehata M, Ju W, Zhang $F$, et al. Prolonged Sinus Pauses upon Termination of Paroxysmal Atrial Fibrillation: Abnormal Right Atrial Electrophysiologic and Electroanatomic Findings. Tex Heart Inst J. 2017;44(2):10714. doi: $10.14503 / \mathrm{THIJ}-15-5493$.

45. Isomoto $S$, Shimizu $A$, Konoe $A$, Kaibara $M$, Tse G, Liu $T$, et al. Tachycardiabradycardia syndrome: Electrophysiological mechanisms and future therapeutic approaches. Int J Mol Med. 2017; 39(3):519-6.

10.3892/ijmm.2017.2877.

46. Centurión O, Shimizu A, Isomoto $S$, Hano $\mathrm{O}$, Hirata $\mathrm{T}$, Konoe $\mathrm{A}$, et al. Incidence and electrophysiological characteristics of the supernormal atrial conduction in humans. J Electrocardiol 1994;27:61-9.

47. Centurión OA, Konoe A, Isomoto S, Hayano M, Kano K. Possible role of supernormal atrial conduction in the genesis of atrial fibrillation in patients with idiopathic paroxysmal atrial fibrillation. Chest 1994; 106:842-7.

48. Shimizu A, Fukatani M, Konoe A, Isomoto $S$, Centurión OA, Yano K. Electrophysiologic effects of new class III antiarrhythmic agent (E-4031) on the conduction and refractoriness of the in-vivo human atrium. Cardiovasc Res 1993; 27:1333-8.

49. Centurión $O A$, Isomoto $S$, Shimizu $A$, Konoe A, Hirata T, Kibara M, et al. Supernormal atrial conduction and its relation to atrial vulnerability and atrial fibrillation in patients with sick sinus syndrome and paroxysmal atrial fibrillation. Am Heart J 1994;128:8895.

50. Jackson LR, Rathakrishnan B, Campbell $K$, Thomas KL, Piccini JP, Bahnson T, et al. Sinus Node Dysfunction and Atrial Fibrillation: A Reversible Phenomenon? Pacing Clin Electrophysiol. 2017;40(4):44250. doi: 10.1111/pace. 13030. 